\title{
A novel pore extraction method for heterogeneous fingerprint images using Convolutional Neural Networks
}

\author{
Ruggero Donida Labatia ${ }^{\mathrm{a}}$, Angelo Genovese ${ }^{\mathrm{a}, * *}$, Enrique Muñoz $^{\mathrm{a}}$, Vincenzo Piuri $^{\mathrm{a}}$, Fabio Scotti $^{\mathrm{a}}$ \\ ${ }^{a}$ Università degli Studi di Milano, Department of Computer Science, 26013 Crema (CR), Italy
}

\begin{abstract}
Most fingerprint recognition systems use Level 1 characteristics (ridge flow, orientation, and frequency) and Level 2 features (minutiae points) to recognize individuals. Level 3 features (sweat pores, incipient ridges and ultra-thin characteristics of the ridges) are less frequently adopted because they can be extracted only from high resolution images, but they have the potential of improving all the steps of the biometric recognition process. In particular, sweat pores can be used for quality assessment, liveness detection, biometric matching in live applications, and matching of partial latent fingerprints in forensic applications. Currently, each type of fingerprint acquisition technique (touch-based, touchless, or latent) requires a different algorithm for pore extraction. In this paper, we propose the first method in the literature able to extract the coordinates of the pores from touch-based, touchless, and latent fingerprint images. Our method uses specifically designed and trained Convolutional Neural Networks (CNN) to estimate and refine the centroid of each pore. Results show that our method is feasible and achieved satisfactory accuracy for all the types of evaluated images, with a better performance with respect to the compared state-of-the-art methods.
\end{abstract}

(c) 2017 Elsevier Ltd. All rights reserved.

\section{Introduction}

Current biometric systems evaluate different kinds of features of fingerprint images for each step of the recognition process. It is possible to classify the fingerprint features into three categories [12, 35]: Level 1 features are characteristics related to the overall ridge flow pattern; Level 2 features consist of the type and coordinates of distinctive points of the ridges, called minutiae points; and Level 3 features are ultra-thin details, such as sweat pores, incipient ridges, and local peculiarities of the ridge edges.

Most automatic fingerprint recognition systems use Level 1 and Level 2 features because they are discernible in images captured with a resolution of at least 500 DPI, which is the standard resolution of most of the current fingerprint recognition systems [9, 35]. Differently, the computation of Level 3 features requires images with higher resolution, captured with at least 800 - 1000 DPI [48].

\footnotetext{
${ }^{* *}$ Corresponding author:

e-mail: angelo.genovese@unimi.it (Angelo Genovese)
}

Recent studies proved that Level 3 features can greatly increase the accuracy of current fingerprint recognition technologies [49-51]. In particular, the number and coordinates of the sweat pores demonstrated to be highly discriminative features $[19,53]$, also in the case of partial fingerprints [2, 3, 34]. These features are particularly suitable for recognition methods dealing with touch-based and touchless samples because the pores are visible in most of the regions of the fingerprint images and their analysis can increase the accuracy of systems with high security requirements such as automated border controls [8]. Differently, the pores are identifiable only in limited regions of good quality latent fingerprint acquisitions. Nevertheless, pore features can be particularly useful for forensic evaluations of latent images, especially in case of partial fingerprints that present a limited number of corresponding minutiae [49]. Moreover, recent liveness detection methods use pore characteristics to distinguish between real and fake fingerprints because most of the fakes do not properly reproduce the real position of all the pores [26]. Another important application that can use pore characteristics is the quality assessment of fingerprint samples [47].

In the literature, there are several studies on the extraction of pore features from specific types of fingerprint samples, cap- 


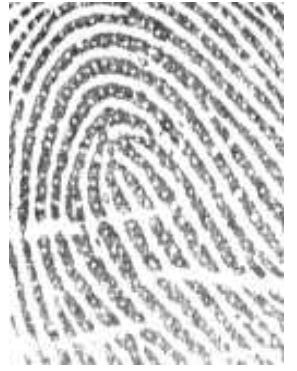

(a)

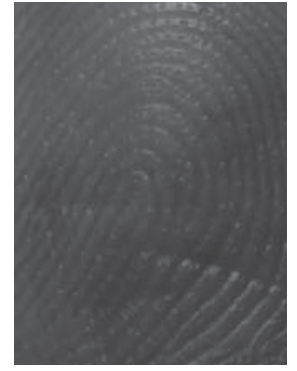

(b)

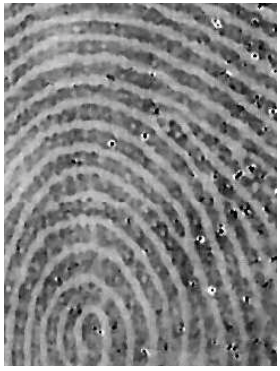

(c)
Fig. 1. An example of fingerprint images acquired using different devices and procedures: (a) image acquired using a touch-based optical device; (b) image acquired using a touchless device; (c) image acquired from a lifted latent impression. The images present different non-idealities that can affect the visibility of the pores: latent fingerprints present a higher level of noise and artifacts with respect to touch-based images, and touchless fingerprint images present a complex background due to the non-uniform color of the skin.

tured live using touch-based optical sensors [19, 53], using touchless devices [15], or lifted from rolled ink impressions [50]. To the best of our knowledge, only one work deals with the extraction of pores from latent impressions [49].

Touch-based fingerprint images, touchless samples, and latent fingerprints present different characteristics. Typically, latent fingerprints present a higher level of noise and artifacts with respect to touch-based images [18]. Touchless fingerprint images present a complex background due to the non-uniform color of the skin $[10,11]$ (Fig. 1). Therefore, methods designed for extracting the pores from a specific type of image can achieve poor results for different kinds of fingerprint samples. Currently, no approach in the literature can extract pores from fingerprint images acquired using different devices.

In this paper, we propose a novel method for extracting the coordinates of the pores from heterogeneous fingerprint images captured using different kinds of devices. Specifically, our method deals with touch-based, touchless, and latent images. To achieve robustness to different kinds of noise, we propose a technique based on Convolutional Neural Networks (CNN). Our method can be divided into several steps: $i$ ) highlight of the position of the pores using a $\mathrm{CNN}$; $i$ ) estimation of the coordinates of the pores; iii) feature extraction from the area around the estimated pores; $i v$ ) discarding of incorrectly detected pores using a CNN.

We performed experiments using more than 23,000 classified pores from three datasets of fingerprint images acquired using high-resolution touch-based sensors, touchless devices, and fingerprints lifted from latent impressions. We acquired the considered touch-based images using an optical sensor with a resolution of 1200 DPI. We captured the touchless samples using a digital single-lens reflex camera and a $100 \mathrm{~mm}$ macro lens, obtaining images captured with a resolution of around 1000 DPI. Among the possible techniques for acquiring latent fingerprints (photographed, lifted, with/without enhancement of substances such as powders, cyanoacrylate or silver nitrate), in this first study we considered images lifted without enhancing substances and acquired using a document scanner with a resolution of 1200 DPI.
We evaluated the performance of our method by analyzing the accuracy of the pore estimation, without applying the pore extraction method in a complete biometric recognition system. An important motivation is that the recognition accuracy is greatly influenced by the used matcher. In fact, different matchers can present a different robustness to falsely estimated pores and falsely non-estimated pores. Furthermore, liveness detection and quality estimation methods also require an accurate pore estimation, without the need for biometric matching. We analyzed the accuracy of the pore estimation by comparing the coordinates of the pores extracted by our approach with those labeled by human operators. Results showed the validity of the proposed methodology, with the majority of the pores correctly extracted. We also compared our method with other well-known methods in the literature, achieving higher accuracy for all the considered types of fingerprint images.

The contributions of the paper are three-fold: i) first, we propose a novel pore extraction technique designed to work with heterogeneous kinds of fingerprint samples; ii) second, we present the first pore extraction method based on $\mathrm{CNN}$; iii) third, we introduce a method that can achieve greater accuracy in extracting the pores with respect to the compared state-ofthe-art techniques.

This paper is organized as follows. Section 2 presents the studies in the literature on the extraction of pores from fingerprint images and briefly overviews CNNs. Section 3 describes the proposed method, while Section 4 presents the experimental protocol and the achieved results. Section 5 concludes the work.

\section{Related work}

This section introduces the pore extraction methods in the literature and briefly overviews CNNs.

\subsection{Extraction of pores from fingerprint images}

Several methods deal with the extraction of Level 3 features, in particular, of the coordinates of sweat pores. There are different pore extraction methods specifically designed for each kind of image, i.e., touch-based images, touchless samples, and latent impressions. These methods aim to increase the accuracy of current techniques for liveness detection, quality assessment, image reconstruction, or biometric recognition.

Liveness detection methods based on characteristics of the pores are attracting the attention of the academic and industrial communities, since it is more difficult to create fake fingers simulating the position of the pores of a real fingerprint, with respect to counterfeiting only the minutia points [26]. Liveness detection methods in the literature evaluate the number of pores $[14,36,45]$, statistical features $[21,31,45]$, Euclidean distance [36], and quality indexes [36, 45].

There also quality assessment methods that analyze porebased features [44, 47] and image reconstruction methods based on the pores extracted from fingerprint images presenting low contrast between the ridges and valleys [41].

The biometric matchers in the literature based on Level 3 features for touch-based images use different techniques to search 
corresponding pairs of points in sets of pore coordinates, such as: Iterative Closest Point (ICP) [19], RANdom SAmple Consensus (RANSAC) [27, 53], Delaunay triangulation [1], or the analysis of Local Binary Patterns (LBP) [33] and other local features $[4,53]$. Some matching strategies use the coordinates and number of pores in conjunction to minutiae features [4951]. There are also methods designed for matching partial fingerprint images $[2,3,34]$, rolled acquisitions [50], or latent fingerprint impressions [49].

The majority of methods for extracting the coordinates of the pores deal with touch-based fingerprint images. These methods use different techniques to estimate the shape of the pores from the samples, such as: Gabor filters [19], watershed segmentation [32], wavelet transforms [1, 19], or morphological operators [5]. To the best of our knowledge, the study presented in [49] is the only attempt of automatic estimation of the pores from latent images. A recent study [15] proposes a pore extraction method for touchless fingerprint images.

All the methods in the literature can only cope with specific kinds of fingerprint images. Differently, the proposed approach can adapt itself to different acquisition scenarios by using computational intelligence techniques, such as CNNs, and achieve better accuracy with respect to other techniques in the literature.

\subsection{Convolutional Neural Networks}

Most of the artificial neural networks in the literature (e.g., feedforward neural networks) consist of layers of neurons that process data in the form of one-dimensional signals. Examples of one-dimensional signals include feature vectors, time series, measurements, and spatial coordinates. Supervised learning procedures allow neural networks to learn from examples and adapt their inner structure to acquire the capability of generalization, with which the neural network is able to approximate the function also in the case of an unknown input signal [17].

In the majority of the cases, a feature extraction step computes the one-dimensional input signals [7] from data with higher dimensionality (e.g., an image). The feature extraction step requires a priori knowledge of the problem to efficiently reduce the dimensionality of the input data, while maintaining the most significant information [25].

CNNs are a particular form of artificial neural networks whose layers have a structure that permits to process data in the form of multi-dimensional arrays, such as images [25]. In particular, the foremost layers of a CNN are multi-dimensional filter banks that process the input image by convoluting it with the corresponding filter. The convolutional layers can use several types of filters to extract the most significant visual features. The subsequent layers convert and aggregate these features into a more abstract representation [24]. Among the advantages of CNNs with respect to neural networks able to process only one-dimensional input signals, there is the fact that CNNs require less prior knowledge of the problem. In fact, CNNs do not require a preliminary feature extraction step because a generic convolutional layer can extract the salient visual features from images depicting a great range of objects and situations. For this reason, researchers use CNNs in different application scenarios, such as object classification [24] or natural language processing [22]. In biometrics, there are recognition methods based on CNNs for different traits, such as: face [6], iris [28, 29], and fingerprint [20]. There are also liveness detection methods bases on CNNs and designed for a wide set of biometric characteristics [37, 39, 46].

\section{The proposed method}

The proposed method can extract Level 3 features from heterogeneous kinds of high-resolution fingerprint images including: images obtained using an optical touch-based scanner, a touchless setup, and latent fingerprints. Taking as input an image with resolution of at least 1000 DPI, our method estimates the coordinates of the centroids of the pores and returns a matrix $P$ of their Cartesian coordinates $(x, y)$.

Fig. 1 shows examples of the heterogeneous kinds of images that can be processed by our method, which present strong differences. Touch-based images, in general, present a good contrast between pores and ridges. Touchless images present areas with different illumination characteristics due to the curvature of the finger. Depending on the illumination, pores may appear as dark blobs in the regions in which the finger is directly illuminated, or bright blobs in the other regions of the finger. Latent images usually have superimposed particles and dust, and therefore have a higher degree of noise, including many artifacts and smooth edges.

The proposed technique has to be robust enough to adapt its parameters to all these possible variations. CNNs are great candidates to deal with such a problem because they can learn the salient features of the pores without needing any assumption on the image characteristics. In our approach, CNNs are used in two steps: first, in a pore detection step, we use CNNs as adaptive filters to enhance the visibility of the pores and remove the ridge-valley pattern of fingerprint images; second, we apply a different kind of CNNs to perform an intelligent refinement to discard the erroneously estimated pores. Fig. 2 shows the schema of the proposed approach, which can be divided into the following steps: i) CNN pore detection; ii) estimation of the coordinates of the candidate pores; iii) filtering and feature extraction; iv) CNN refinement.

\subsection{CNN for pore detection $\left(C N N_{D}\right)$}

This section presents the $\mathrm{CNN}$ used to detect the pores, named $C N N_{D}$, and the related training process.

$C N N_{D}$ takes as input a gray-scale image $I$ and returns the image $I_{C N N}$, in which the pores are enhanced and the ridge pattern is removed. The goal of the network is to compute an image $I_{C N N}$ in which the intensity of the centers of the pores is equal to 1 and that of the other pixels is equal to -1 .

To speed up the convergence of the learning process, we apply a simple preprocessing to $I$ before using $C N N_{D}$. We perform a min-max normalization followed by the subtraction of the mean intensity of the image.

\subsubsection{Architecture}

Differently from the CNN configurations most commonly used in the literature for classification problems, the proposed 


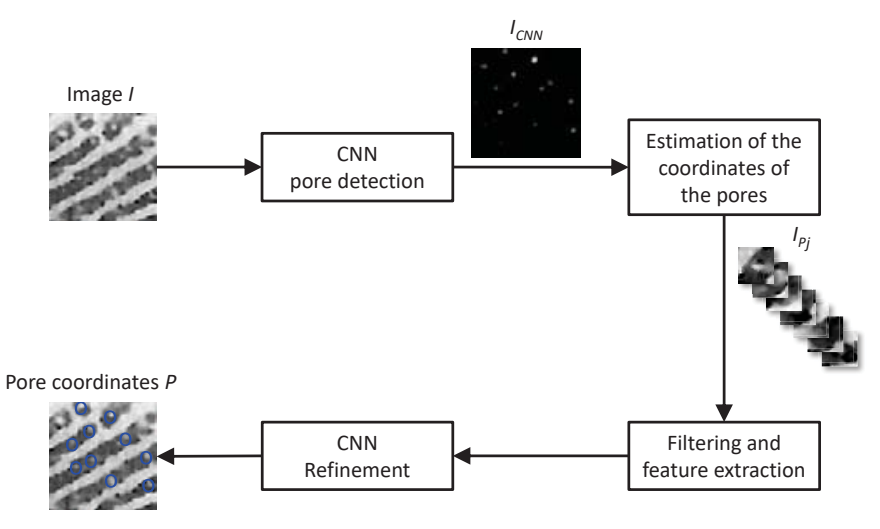

Fig. 2. Schema of the proposed method. First, a CNN performs pore detection, enhancing the visibility of the pores and removing the ridge pattern and the background. Then, the coordinates of the centers of the pores are estimated. Later, for each found pore, different features are extracted. In the last step, a $\mathrm{CNN}$ refines the results discarding the incorrectly detected pores.

method does not use a final fully connected Softmax layer. In most of the CNNs used in classification problems, the size of the images decreases after each layer and the last layer provides the class of the image. In contrast, our approach uses CNNs as adaptive filters to enhance the pores and remove the ridge pattern. As a result, $C N N_{D}$ keeps the size of the image constant from layer to layer and the final layer returns an image $I_{C N N}$ with the same size of the input image $I$.

The proposed CNN architecture uses only convolutional layers and pooling layers. Each convolutional layer computes an output $y$ by taking as input a map $x$ and convolving it with a bank of $K$ two-dimensional filters $f$, using biases $b$. Here

$$
x \in \mathbb{R}^{H \times W}, f \in \mathbb{R}^{H^{\prime} \times W^{\prime}}, y \in \mathbb{R}^{H^{\prime \prime} \times W^{\prime \prime}},
$$

where $H$ and $W$ are the height and width dimensions, respectively. In the basic configuration of the convolutional layer, for each coordinate $(i, j)$, the output is computed as follows:

$$
y_{i^{\prime} j^{\prime}}=b+\sum_{i^{\prime}=1}^{H^{\prime}} \sum_{j^{\prime}=1}^{W^{\prime}} f_{i^{\prime} j^{\prime}} \times x_{i^{\prime \prime}+i^{\prime}-1, j^{\prime \prime}+j^{\prime}-1} .
$$

In this layer, we perform a padding of constant step $\left(P_{h}, P_{w}\right)$ of the input $x$ along the $H$ and $W$ axes, respectively. Specifically, we perform top-bottom-left-right padding. We also use subsampling strides $\left(S_{h}, S_{w}\right)$, as follows:

$$
y_{i^{\prime} j^{\prime}}=b+\sum_{i^{\prime}=1}^{H^{\prime}} \sum_{j^{\prime}=1}^{W^{\prime}} f_{i^{\prime} j^{\prime}} \times x_{S_{h}\left(i^{\prime \prime}-1\right)+i^{\prime}-P_{h}, S_{w}\left(j^{\prime \prime}-1\right)+j^{\prime}-P_{w}} .
$$

The pooling layers utilize the max-pooling operator, which reduces the feature space by computing the maximum response of each feature in a $H^{\prime} \times W^{\prime}$ patch, as follows:

$$
y_{i^{\prime} j^{\prime}}=\max _{1 \leq i^{\prime} \leq H^{\prime}, 1 \leq j^{\prime} \leq W^{\prime}} x_{i^{\prime \prime}+i^{\prime}-1, j^{\prime \prime}+j^{\prime}-1},
$$

resulting in an output of size $y \in \mathbb{R}^{H^{\prime \prime} \times W^{\prime \prime}}$.

Fig. 3 shows the architecture of $C N N_{D}$. The network has 5 layers, including 3 convolutional layers and 2 max pooling layers. The first convolutional layer consists of 5 filters with size $a_{1} \times a_{1}$ pixels, striding equal to $(1,1)$, and padding equal to $\left(\frac{a-1}{2}, \frac{a-1}{2}\right)$. This convolutional layer is followed by a maxpooling with a kernel of $3 \times 3$ pixels. The second convolution layer contains 15 filters of size $a_{2} \times a_{2}$ pixels, striding equal to $(1,1)$, and padding equal to $\left(\frac{a-2}{2}, \frac{a-2}{2}\right)$. This convolutional layer is followed by a max-pooling with a kernel of $3 \times 3$ pixels. The last convolutional layer includes a single filter of size $a_{3} \times a_{3}$ pixels, striding equal to $(1,1)$, and padding equal to $\left(\frac{a-3}{2}, \frac{a-3}{2}\right)$. The number of layers, the size of the convolution kernels and max-pooling kernels and the stride have been tuned empirically.

\subsubsection{Training}

The objective of the training process is to obtain a CNN that provides an output of at least 1 for the pixels representing the centroid of a pore, and at most -1 for the pixels far from any pore. To achieve this goal, we use a training dataset composed of multiple labeled images. Human experts labeled each pixel of each image belonging to this dataset. The labels are equal to: 1 for the pixels representing the centroid of a pore; 0 for the pixels lying in a circle with radius $r_{p}$ and center in the centroid of a pore $\left(r_{p}=5\right.$ pixels for touch-based images, $r_{p}=4$ pixels for touchless samples, and $r_{p}=7$ pixels for latent fingerprints); and -1 for all the other pixels.

We train $C N N_{D}$ using the well-known algorithm based on stochastic gradient descent with momentum [16]. We initialize the weights of the filters used by $C N N_{D}$ by applying a random sampling from a Gaussian distribution with zero mean and 0.01 standard deviation. $C N N_{D}$ is trained for 500 epochs, using the learning rates 5,5 and 0.0005 for touch, touchless, and latent fingerprints, respectively. The batch size is set to the number of images in the learning database.

\subsection{Estimation of the coordinates of the pores}

This step estimates a matrix $P$ of the Cartesian coordinates of the centers of the pores from $I_{C N N}$. First, we set to -1 the pixels of the output image $I_{C N N}$ with negative intensity, because they represent regions of the image far from the centers of the pores. Second, we compute a binary map of the pores $B$ by thresholding $I_{C N N}$ with the Otsu's algorithm [40]. Third, we compute the coordinates of each pore $P_{i}=\left(x_{i}, y_{i}\right)$ as the centroid $\left(x_{i}, y_{i}\right)$ of each white region $i$ of $B$. We discard the white regions with area equal or less than an empirically tuned threshold $t_{a}$.

\subsection{Filtering and feature extraction}

For each pore $P_{i}$, we compute a four channel image $F_{i}$ of size $21 \times 21$ pixels, representing the values of 4 features computed pixelwise from the local region centered in $P_{i}$. The four channels are obtained as follows: $i$ ) the local intensity of $I$; $i$ i) the local intensity of $I_{C N N}$; iii) the local intensity of the binary ridge map $R ; i v$ ) the local intensity of the radial symmetry image $I_{C} . R$ provides information on the ridge-valley pattern of the fingerprint to the $\mathrm{CNN}$. To compute $R$, we apply the algorithm described in [23]. $I_{C}$ enhances the visibility of regions with high radial symmetry of $I_{C N N}$, thus providing information on the presence of circular shapes to the CNN. We compute $I_{C}$ by applying the fast RST method proposed in [30] to $I_{C N N}$ using radii $=[1,2, \ldots, 9]$. 


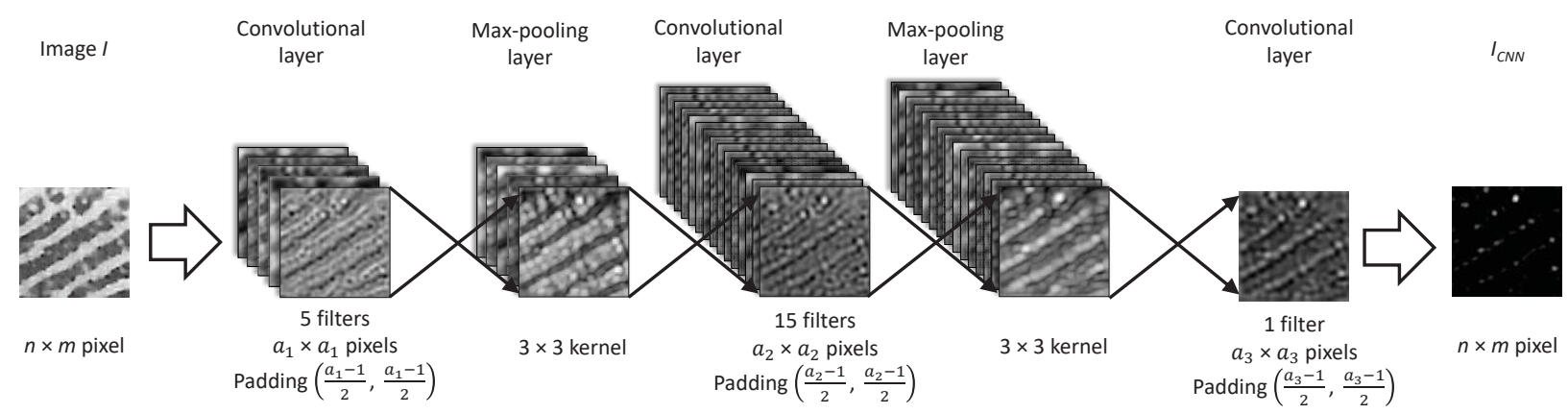

Fig. 3. Schema of the proposed CNN used for pore detection $\left(C N N_{D}\right)$. The goal of the network is to compute an image $I_{C N N}$ in which the intensity of the pores is equal to 1 and all the other pixels are equal to 0 . The network is composed of 5 layers, including three convolutional layers and two max-pooling layers.

\section{4. $C N N$ for refinement $\left(C N N_{R}\right)$}

This step aims to remove possible errors from the matrix $P$ of candidate pores. To this end, we propose a CNN, named $C N N_{R}$, that searches erroneously estimated pores. For each pore $P_{i}, C N N_{R}$ analyzes the local values of the feature image $F_{i}$ extracted from a local region of $I$ surrounding $P_{i} . C N N_{R}$ returns a binary value 1 for the coordinates considered as true pores, and 0 for false pores.

Also in this case, to speed up the convergence of the learning process, we perform a simple preprocessing to each image $F_{i}$ before using $C N N_{R}$. We perform a min-max normalization followed by the subtraction of the mean intensity of the image.

\subsubsection{Architecture}

In contrast with $C N N_{D}, C N N_{R}$ uses a general architecture for classification that decreases the size of the image after each layer. Fig. 4 shows the architecture of $C N N_{R}$.

The proposed CNN architecture uses convolutional layers, pooling layers, a ReLu (Rectified Linear Units) layer and a final Softmax loss layer. Convolutional and pooling layers are detailed in Section 3.1.1. The ReLu layer uses a non-saturating activation fuction

$$
y_{i}=\max \left(0, x_{i}\right),
$$

while the Softmax classifier computes its output as

$$
y_{j}=e^{x_{j}} /\left(\sum_{i^{\prime}=1}^{n} e^{x_{i}}\right)
$$

where $n$ is the number of inputs to the neuron.

$C N N_{R}$ is composed of 8 layers, including 4 convolutional layers, 2 max-pooling layers, a ReLu layer, and a Softmax classification layer. The first convolutional layer consists of 10 filters of size $a_{4} \times a_{4}$ pixels, striding equal to $(1,1)$, and no padding. This convolutional layer is followed by a max-pooling layer with a kernel equal to $3 \times 3$ pixels. The second convolutional layer consists of 25 filters of size $a_{5} \times a_{5}$ pixels, striding equal to $(1,1)$, and no padding. This convolutional layer is followed by a max-pooling layer with a kernel equal to $3 \times 3$ pixels. The third convolutional layer consists of 250 filters of size $a_{6} \times a_{6}$ pixels, striding equal to $(1,1)$, and no padding. This convolutional layer is followed by a ReLu layer. The last convolutional layer consists of two filters of size $a_{7} \times a_{7}$ pixels.
The last layer is a fully-connected Softmax classifier returning a binary value representing if the pore $P_{i}$ can be considered as properly estimated. The number of layers, the size of the convolution kernels and max-pooling kernels, and the stride have been tuned empirically.

\subsubsection{Training}

The training database consists of the images $F_{i}$ obtained for each candidate pore $P_{i}$ computed from each image $I$ used to train $C N N_{D}$. The label of each image $F_{i}$ is equal to 1 if it corresponds to a pore, and 0 otherwise.

We use the stochastic gradient descent with momentum algorithm [16] to train $C N N_{R}$. We initialize the weights of the filters using a random sampling from a Gaussian distribution with zero mean and 0.01 standard deviation. Each CNN is trained for 400 epochs, using the learning rate 0.0005 for touchless and latent fingerprints. No refinement is necessary for touch-based fingerprints. The batch size is set to 500 .

\section{Experiments and discussion}

This section describes the experimental protocol, the used datasets, the achieved results, and the computational time of our approach. We evaluated the accuracy of the proposed approach on sets of touch-based images, samples acquired using touchless sensors, and latent fingerprints. We tested our method using more than 23,000 classified pores, proving the validity of the proposed methodology, with the majority of the pores correctly extracted. We also compared the proposed approach with other well-known methods in the literature. In all the considered types of fingerprint images, our approach obtained the highest accuracy, with a reasonable computational cost.

\subsection{Experimental protocol}

\subsubsection{Datasets}

We used datasets of fingerprints acquired with different modalities, specifically: sets of touch-based images, samples acquired by means of a touchless sensor, and latent fingerprints. For each dataset, human operators created a ground truth for our experiments by estimating the center coordinates of all the pores. Fig. 5 shows some examples of the used images.

Fig. 5 shows examples of images used to evaluate the accuracy of our pore detection method. These images present a 


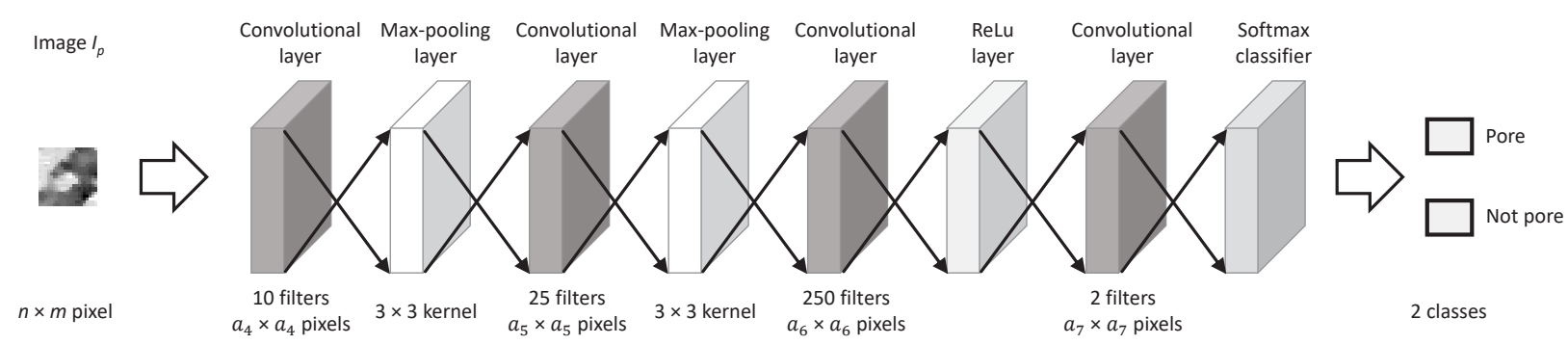

Fig. 4. Schema of the proposed $\mathrm{CNN}$ for refinement $\left(C N N_{R}\right)$. The goal of the network is to determine if a candidate pore is a real pore or not. The network is composed of 8 layers, including four convolutional layers, two max-pooling layers, one ReLu layer and a Softmax classifier.

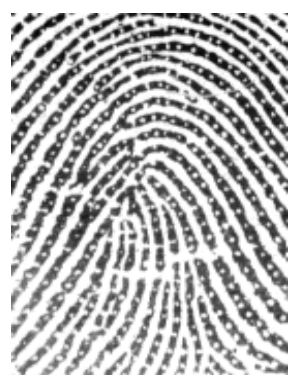

(a)

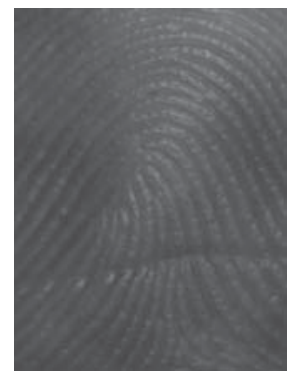

(b)

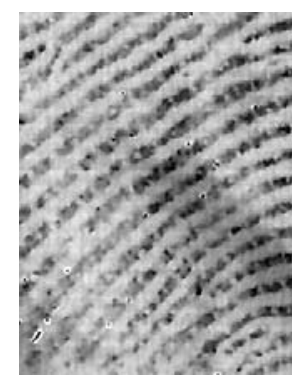

(c)
Fig. 5. Examples of used images: (a) touch-based image from DB Touchbased; (b) touchless image from DB Touchless; (c) latent image from DB Latent. It is possible to observe that, while the pores are visible in all three images, the images present a great variability.

great variability: touch-based fingerprint images present high contrast between the ridges and pores; touchless samples have a complex background, reflections, and pores with darker or brighter intensity with respect to the ridges; latent fingerprints present artifacts due to the acquisition process and low contrast between ridges and pores.

We tested our method using the following datasets:

- DB Touch-based: this dataset contains the 30 labeled images belonging to the PolyU High-Resolution-Fingerprint (HRF) database [53]. The labels represent the Cartesian coordinates of 12767 pores. The images have a resolution of 1200 DPI, have size $320 \times 240$ pixels, and depict the central region of the finger. The used acquisition device is the optical touch-based sensor described in [48]. The fingerprint images as well as the labeled coordinates of the pores are public and different methods in the literature [27, 53] have used them to evaluate their performance.

- DB Touchless: we collected this dataset in our laboratory [15]. It consists of 44 touchless fingerprint images captured from 22 fingers by using a Canon 6D camera with a $100 \mathrm{~mm}$ macro lens. The images have a resolution of $\approx 1000 \mathrm{DPI}$, have size $250 \times 250$ pixels, and depict the central region of the finger. We labeled the coordinates of the pores of each image, for a total of 9,143 pores. Fig. 6 (b) shows an example of a touchless sample with labeled pores, comparing it with a touch-based image of the same finger (Fig. 6 (a)). To the best of our knowledge, this is the only dataset of touchless fingerprint images that present sufficient resolution and sharpness for evaluating Level 3 features such as pores.

- DB Latent: we collected this dataset in our laboratory. It consists of 36 latent fingerprints of 22 fingers. We acquired the latent fingerprint using forensic procedures and digitalized the images using a document scanner. The images have a resolution of 1200 DPI, with size $320 \times 240$ pixels. We labeled the coordinates of the pores of each image, for a total of 1,785 pores. Fig. 6 (e) shows an example latent fingerprint with labeled pores, comparing it with a touchbased image of the same finger (Fig. $6(d)$ ). To the best of our knowledge, there is only a study in the literature that deals with pore extraction from latent samples [49]. This study used images from the ELFT-EFS Public Challenge Dataset [18] and a set of latent images pertaining to the West Virginia University. However, these datasets are not publicly available anymore. Another dataset of latent fingerprint images with high resolution is the IIIT-D Latent Fingerprint Database [43]. Nevertheless, this dataset is not suitable for our tests because it is composed of images of unknown and inconstant resolution, acquired using a digital camera.

\subsubsection{Evaluation procedure}

For each image of the used datasets, we applied our method for estimating of the coordinates of the sweat pores. We tested the method using a k-fold validation strategy [13], with $k=5$. In particular, we used 3 folds for training, 1 fold for validation and 1 fold for testing.

We used two figures of merit: the true detection rate $\left(R_{T}\right)$ and the false detection rate $\left(R_{F}\right)$ [52]. $R_{T}$ represents the ratio of the number of detected real pores to the number of all true pores present in the image. $R_{F}$ indicates the ratio of the number of falsely detected pores to the total number of detected pores. The optimal values for $R_{F}$ and $R_{T}$ are zero and one, respectively.

We consider that a pore is correctly detected if its Euclidean distance from the coordinates of a pore labeled by a human operator is equal or less than $d$ pixels. We define $d=r w / 2$, where $r w$ is the average ridge width in the images of the considered dataset.

To compare the performance of the proposed method with other techniques in the literature, we implemented the following algorithms:

- Inversion: this technique uses basic image processing operators. First, it creates a binary image $I_{B}$ by binarizing 


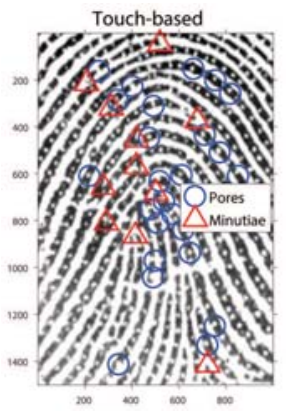

(a)

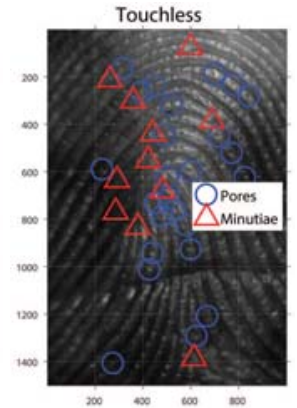

(b)

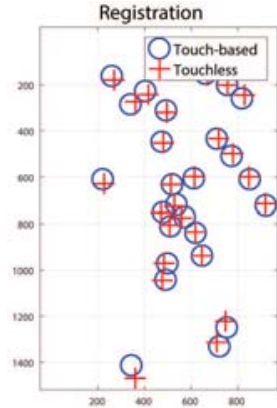

(c)

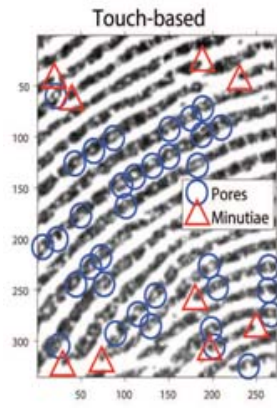

(d)

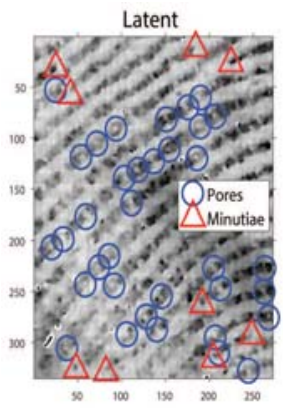

(e)

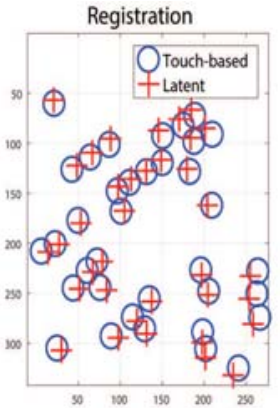

(f)

Fig. 6. An example of the ground truth extracted from an image of DB Touchless and DB Latent: (a,d) touch-based images; (b) touchless sample; (c) registration of the extracted pores present in (a) and (b); (e) latent image; (f) registration of the extracted pores present in (d) and (e). The registration is based on an ICP-based algorithm [38]. Many pores are visible in both images (only a subset of pores identified by the supervisor has been plotted to make the plot chart more readable).

the input fingerprint image $I$, using a threshold obtained with Otsu's method [40]. The algorithm then computes two binary images, $I_{B 1}$ and $I_{B 2}$, obtained by removing the 8 -connected regions with area smaller or equal to 40 pixels and an area equal to 1 pixels from $I_{B}$, respectively. It then creates a binary image $I_{P}$, which represents the pores, as $I_{P}=\operatorname{XOR}\left(I_{B 1}, I_{B 2}\right)$. Finally, the algorithm computes the matrix of the coordinates of the pores $P$ by estimating the centroids of the 8-connected regions of $I_{P}$.

- Gabor filters: the technique is the pore extraction algorithm described in [19], which fuses the information obtained by applying Gabor filters and Mexican Hat filters.

- Neural classifiers: the method is described in [15], is based on neural classifiers, and is specifically designed for touchless images.

\subsection{Performance of the proposed method}

\subsubsection{Results for DB Touch-based}

In touch-based images the pores are clearly visible, the contrast between ridges and pores is high, and the number of artifacts is low. For this reason, it was not necessary to use the full architecture that includes both $C N N_{D}$ and $C N N_{R}$ to obtain satisfactory results. In particular, we could omit the refinement step of $\left(\mathrm{CNN}_{R}\right)$, and use only a simplified version of $\left.\mathrm{CNN}_{D}\right)$ for estimating the coordinates of the pores. The CNN includes three layers: a convolutional layer composed of 30 filters of size $5 \times 5$ pixels, a max-pooling layer with a kernel of $3 \times 3$ pixels, and a convolutional layer composed of 1 filter of size $5 \times 5$ pixels.

Table 1 reports the accuracy achieved by the proposed approach for DB Touch-based. The obtained results are compared with those presented in [52], which are related to the methods developed by Ray et al. [42], Gabor filters [19], Adaptive DoG, and DAPM [52]. To perform this comparison, we computed the mean and standard deviation of the $R_{T}$ and $R_{F}$. The results reported in [52] refer to a subset of 24 unknown images of DB Touch-based, while we tested our method using all the 30 images of DB Touch-based. Our method achieved better accuracy with respect to the compared state-of-the-art techniques. Only the algorithm presented in [49] obtained similar performance.
Table 1. Average performance metrics in percentage and standard deviation (in parenthesis) for DB Touch-based.

\begin{tabular}{cccccc}
\hline $\begin{array}{c}\text { Ray et al. } \\
\text { [42] }\end{array}$ & $\begin{array}{c}\text { Gabor filters* } \\
\text { [19] }\end{array}$ & $\begin{array}{c}\text { Adapt. DoG* } \\
\text { [52] }\end{array}$ & $\begin{array}{c}\text { DAPM }^{*} \\
\text { [52] }\end{array}$ & CNN $_{D}$ \\
\hline$R_{T}$ & $60.6(11.9)$ & $75.9(7.5)$ & $80.8(6.5)$ & $84.8(4.5)$ & $84.69(7.81)$ \\
$R_{F}$ & $30.5(10.9)$ & $23.0(8.2)$ & $22.2(9.0)$ & $17.6(6.3)$ & $15.31(6.20)$ \\
\hline
\end{tabular}

* These results were obtained using a subset of 24 images of the dataset used in our experiments, which contains a total of 30 images. However, no information regarding which 24 images were chosen was found.

Table 2. Average performance metrics in percentage and standard deviation (in parenthesis) for DB Touchless.

\begin{tabular}{ccc}
\hline & Neural classifiers [15] & $\mathbf{C N N}_{D}+\mathbf{C N N}_{R}$ \\
\hline$R_{T}$ & $22.9(5.6)$ & $51.8(8.3)$ \\
$R_{F}$ & $16.3(7.7)$ & $11.7(6.6)$ \\
\hline
\end{tabular}

For completeness, we implemented the technique described in [49] and compared its performance with that of our method for all the images of DB Touch-based. Fig. 7 presents the average $1-R_{F}$ obtained by the compared pore extractors for different values of $d$. This graph shows that the proposed approach properly detected a larger amount of pores with respect to the compared method.

Fig. 8 (a) presents a visual example of the pores estimated by the proposed approach. This figure shows a relevant number of detected pores corresponding to the labeled ones. We obtained similar results for all the images belonging to DB Touch-based. Since Jain et al. [19] demonstrated that about 20-40 pores are sufficient to assess the identity of an individual, we can infer that our pore extraction method can be successfully applied to perform recognition tasks with touch-based images.

\subsubsection{Results for DB Touchless}

Touchless images are the most difficult to analyze, because of the variability in illumination and pore reflectance. Table 2 presents the results obtained for DB Touchless. We compared the results of our method with those achieved by the technique proposed in the work [15], which is the only pore extractor in literature designed for touchless fingerprint images. To perform this comparison, we computed the mean and standard deviation of the $R_{T}$ and $R_{F}$. Table 2 shows that our method outperforms 


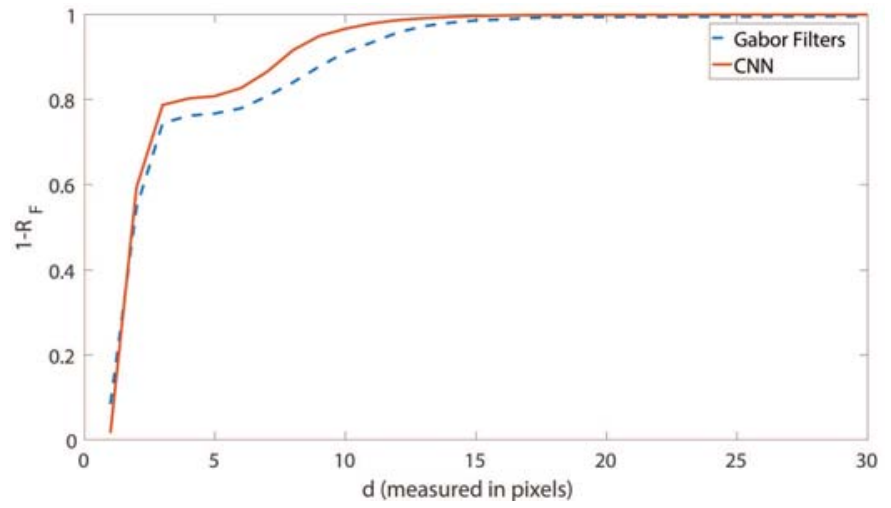

Fig. 7. Average $1-R_{F}$ obtained by each approach on DB Touch for different values of $d$. The displayed curves present the results obtained with Gabor filters [19] and the proposed approach based on CNN. Our method obtained the greatest pore estimation accuracy.

Table 3. Average performance metrics in percentage and standard deviation (in parenthesis) for DB Latent.

\begin{tabular}{cccc}
\hline & Gabor filters [19] & Inversion & $\mathbf{C N N}_{D}+\mathbf{C N N}_{R}$ \\
\hline$R_{T}$ & $32.9(29.9)$ & $34.8(15.0)$ & $52.7(14.5)$ \\
$R_{F}$ & $79.8(17.8)$ & $84.3(10.9)$ & $24.0(11.2)$ \\
\hline
\end{tabular}

Table 4. Average performance metrics in percentage and standard deviation (in parenthesis) for 28 selected areas of $100 \times 100$ pixels of images in DB Latent.

\begin{tabular}{cccc}
\hline & Gabor filters [19] & Inversion & $\mathbf{C N N}_{D}+\mathbf{C N N}_{R}$ \\
\hline$R_{T}$ & $45.3(19.2)$ & $46.0(27.4)$ & $63.8(16.0)$ \\
$R_{F}$ & $44.9(23.3)$ & $58.2(30.1)$ & $17.6(11.7)$ \\
\hline
\end{tabular}

the work in [15] in both figures of merit. These results indicate that, also for touchless images, our CNN-based method can find more pores with fewer errors with respect to state-of-theart techniques. Fig. 8 (b) presents an example of pores retrieved by the proposed approach. Also in the case of touchless samples, the number of pores retrieved per image can be sufficiently high to apply recognition methods based on the coordinates of the pores.

\subsubsection{Results for DB Latent}

Table 3 reports the results obtained for DB Latent. We compared our method with the technique presented in [19] and Inversion. To assess the performance, we computed the mean and standard deviation of the $R_{T}$ and $R_{F}$. The results confirmed that latent fingerprints present additional challenges with respect to touch-based images because all techniques achieved worse performance with respect to the results reported in Table 1. Nonetheless, the proposed method based on CNN showed a higher adaptability with respect to the compared techniques, achieving better values of $R_{T}$ and $R_{F}$. Fig. 8 (c) shows an example of the output of the pore extractor, in which the number of pores retrieved per image is sufficiently high to perform recognition tasks using the position of the pores.

One of the main problems of latent fingerprints is that there are several areas that do not show any pore, which increases the complexity of pore extraction. To study the performance of the method with portions of the images that contain a significant number of pores, we selected a subset of 28 images of size $100 \times$ 100 pixels. Table 4 presents the results achieved by our method and by the method presented in [19] and Inversion. All methods obtained a better accuracy with respect to the results reported in Table 1, although our method achieved the best performance.

\subsubsection{Accuracy of refinement methods}

The refinement based on $\mathrm{CNN}_{R}$ is an important step of our pore extraction method. In this section, we study the impact of this step on the overall method accuracy. As a test case, we chose latent fingerprints. We compared the proposed $\mathrm{CNN}_{R}$ with the following techniques:

- Simple refinement: the 4-connected regions of binary map of the pores $B$ with area greater than 50 pixels, area less than 2 pixels, or high ratio between the major and minor axis (greater than 2) are discarded.

- Circle refinement: this algorithm discards pores with low radial symmetry. It discards a candidate pore $P_{i}$ of coordinates $\left(x_{i}, y_{i}\right)$ if $I_{C}\left(x_{i}, y_{i}\right)<0.12$.

- CI-based refinement: we use this prefix for methods based on computational intelligence (CI) that exploit features extracted from the local regions surrounding each candidate pore $P_{i}$. The methods considers local regions of $11 \times 11$ pixels centered $\mathrm{i}\left(x_{i}, y_{i}\right)$. The extracted features included: average intensity and standard deviation of the local region of $I$, average and standard deviation of the local region of $I_{C N N}$, the quantiles $0.1,0.5$ and 0.9 of the local region of $I$, the quantiles $0.1,0.5$ and 0.9 of the local region of $I_{C N N}$, and the quantiles $0.1,0.5$ and 0.9 of the local region of $I_{C}$. We used the following CI techniques:

- k-nearest neighbors $(\mathrm{kNN})$, with different numbers of neighbors;

- naive Bayes classifier;

- Support Vector Machine with polynomial kernel function.

Table 5 presents the obtained results, showing that $\mathrm{CNN}_{R}$ achieved the best performance. In particular, these results show that $\mathrm{CNN}_{R}$ removed a much higher number of falsely estimated pores with respect to the compared refinement techniques.

\subsubsection{Computational time analysis}

In this section, we analyze the time required by our pore extraction method. We executed the tests using a PC with $3.7 \mathrm{GHz}$ Intel (R) Xeon (R) E5-1620 v2 CPU, RAM 16 GB and NVIDIA (R) Quadro (R) K4000 3GB GPU. The operating system was Windows 7 professional 64 bit. All methods were implemented using Matlab.

For touch-based images, the pore extraction process $\left(C N N_{D}\right.$ and the estimation of the coordinates of the pores) took about 0.2 s per image. The GPU parallelization of $C N N_{D}$ reduced the execution time of about $5 \mathrm{~ms}$. The impact of GPU parallelization is not very significant because the most time consuming task is the estimation of the coordinates of the pores. We 


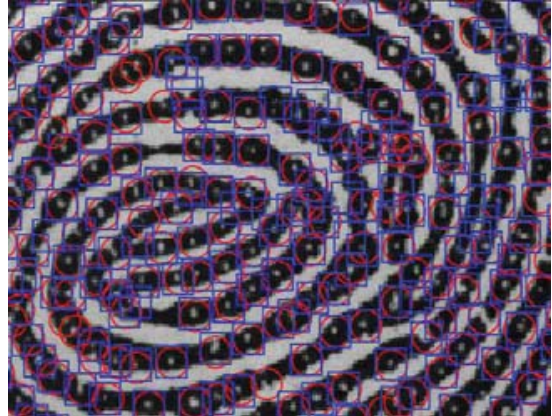

(a)

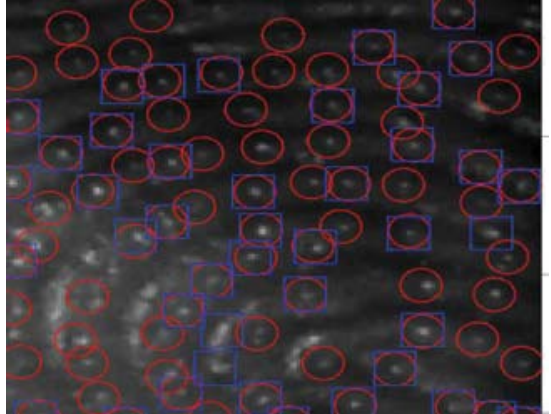

(b)

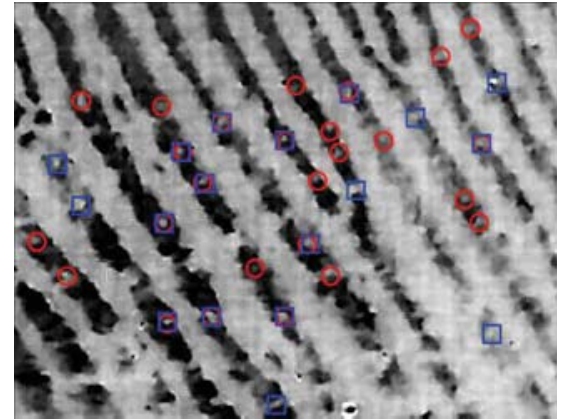

(c)

Fig. 8. Examples of pores extracted by the proposed detector in an image from (a) DB Touch-based, (b) DB Touchless, (c) DB Latent. Red circles indicate pores labeled by the supervisors, while blue squares indicate the pores obtained by our method.

Table 5. Average performance metrics in percentage and standard deviation (in parenthesis) for DB Latent using different refinement schemes.

\begin{tabular}{ccccccccc}
\hline & $\begin{array}{c}\mathrm{CNN}_{D}+ \\
\mathrm{Simple}\end{array}$ & $\begin{array}{c}\mathrm{CNN}_{D}+ \\
\mathrm{Circle}\end{array}$ & $\begin{array}{c}\mathrm{CNN}_{D}+ \\
\mathrm{CI}-\mathrm{kNN1}\end{array}$ & $\begin{array}{c}\mathrm{CNN}_{D}+ \\
\mathrm{CI}-\mathrm{kNN5}\end{array}$ & $\begin{array}{c}\mathrm{CNN}_{D}+ \\
\mathrm{CI}-\mathrm{kNN10}\end{array}$ & $\begin{array}{c}\mathrm{CNN}_{D}+ \\
\text { CI-Bayes }\end{array}$ & $\begin{array}{c}\mathrm{CNN}_{D}+ \\
\mathrm{CI}^{+} \mathrm{SVM}\end{array}$ & $\begin{array}{c}\mathrm{CNN}_{D}+ \\
\mathrm{CNN}_{R}\end{array}$ \\
\hline$R_{T}$ & $89.6(3.9)$ & $72.1(16)$ & $46.4(10.4)$ & $40.8(11.3)$ & $32.4(12.1)$ & $52.1(17.0)$ & $49.2(14.6)$ & $52.7(14.5)$ \\
$R_{F}$ & $80.8(6.0)$ & $52.2(14.3)$ & $54.2(8.8)$ & $44.2(10.22)$ & $35.9(12.4)$ & $40.3(15.4)$ & $52.6(12.5)$ & $24.0(11.2)$ \\
\hline
\end{tabular}

Note: Simple $=$ Simple refinement; Circle $=$ Circle refinement; CI-kNN $=$ CI-based refinement using k-nearest neighbors $(\mathrm{kNN})$; CI-Bayes $=$ CI-based refinement using naive Bayes classifier; CI-SVM = CI-based refinement using Support Vector Machine with polynomial kernel function; CNN-R = CNN Refinement.

also implemented one of the methods used in the state-of-theart method Gabor filters [19], which required around $0.8 \mathrm{~s}$ to extract the pores from each image. In comparison, our method is around 4 times faster. This is mainly due to the absence of a preprocessing step that required around $0.7 \mathrm{~s}$ per image for the Gabor filters method.

For touchless images, the use of two consecutive CNNs increased the execution time. The analysis of touchless images required around $0.7 \mathrm{~s}$ per image, divided into application of $C N N_{D}(0.1 \mathrm{~s})$, estimation of the coordinates of the pores and pore image creation $(0.5 \mathrm{~s})$ and application of $C N N_{R}(0.1 \mathrm{~s})$. In comparison, the method based on neural classifiers presented in [15] took around $0.8 \mathrm{~s}$ per image, which can be divided into pore detection $(0.3 \mathrm{~s})$, pore feature extraction $(0.5 \mathrm{~s})$ and neural post-processing $(0.01 \mathrm{~s})$. The $\mathrm{CNN}$-based method is faster, mainly because $C N N_{D}$ is more precise than the first step of the method presented in [15] and extracts less candidate pores.

For latent fingerprints, our method required around $0.3 \mathrm{~s}$. As with touchless images, the execution time can be divided into application of $C N N_{D}(0.02 \mathrm{~s})$, estimation of the coordinates of the pores and pore image creation (0.2 s) and application of $C N N_{R}(0.1 \mathrm{~s})$. Gabor filters method, in comparison, took around $0.2 \mathrm{~s}$. In this case, the CNN based method is slower, due to the refinement step. Nonetheless, considering the improvement in pore detection accuracy, we believe that the increase in computational time is deserved.

We think that the computational cost of our method is acceptable since Matlab is a prototype-oriented and non-optimized environment. We expect that the use of compiled languages, such as $\mathrm{C} / \mathrm{C}++$, can reduce the processing time, obtaining real-time performance.

\section{Conclusions and future work}

In this work, we proposed a novel method for the extraction of Level 3 features. In particular, our method is designed for estimating the coordinates of sweat pores from heterogeneous fingerprint images, including touch-based, touchless and latent samples. Given the different characteristics of the analyzed images, which include different levels of noise, artifacts, and quality of illumination conditions, it was necessary to design a very flexible pore extraction method. Convolutional Neural Networks (CNN) provide a suitable solution to this problem because they can adapt their parameters to different conditions by learning from examples. Furthermore, they do not require any assumption on the characteristics of the input images.

In the presented work, we performed tests on more than 23, 000 classified pores. We used three image datasests composed of 30 touch-based images, 44 touchless samples, and 36 latent fingerprints. Results showed the validity of the proposed methodology, with the majority of the pores correctly extracted. We compared our results with those of other well-known methods in the literature. Our method achieved higher accuracy for all the considered types of fingerprint images.

Future work should regard the design of novel matching algorithms based on features related to the pores and able to deal with heterogeneous fingerprint images. In addition, the proposed approach could be exploited to design innovative and more accurate liveness detection algorithms able to work with samples acquired using different technologies.

\section{Acknowledgements}

This work was supported in part by: the EC within the 7FP under grant agreement 312797 (ABC4EU); the EC within 
the H2020 program under grant agreement 644597 (ESCUDOCLOUD); and the Italian Ministry of Research within PRIN 2015 project COSMOS (201548C5NT).

\section{References}

[1] Abhyankar, A., Schuckers, S., 2010. Towards integrating level-3 features with perspiration pattern for robust fingerprint recognition, in: Proc. of the 17th IEEE Int. Conf. on Image Processing (ICIP), pp. 3085-3088.

[2] Agrawal, P., Kapoor, R., Agrawal, S., 2014. A hybrid partial fingerprint matching algorithm for estimation of Equal Error Rate, in: Proc. of the Int. Conf. on Advanced Communication Control and Computing Technologies (ICACCCT), pp. 1295-1299.

[3] Chen, Y., Jain, A.K., 2007. Dots and incipients: Extended features for partial fingerprint matching, in: Proc. of the Biometrics Symp., pp. 1-6.

[4] Cui, J., Ra, M.S., Kim, W.Y., 2014. Fingerprint pore matching method using polar histogram, in: Proc. of the 18th IEEE Int. Symp. on Consumer Electronics (ISCE), pp. 1-2.

[5] Da Silva Teixeira, R.F., Leite, N.J., 2013. On adaptive fingerprint pore extraction, in: Kamel, M., Campilho, A. (Eds.), Image Analysis and Recognition. Springer Berlin Heidelberg. volume 7950 of Lecture Notes in Computer Science, pp. 72-79.

[6] Ding, C., Tao, D., 2015. Robust face recognition via multimodal deep face representation. IEEE Trans. on Multimedia 17, 2049-2058.

[7] Donida Labati, R., Genovese, A., Muñoz, E., Piuri, V., Scotti, F., Sforza, G., 2016a. Computational intelligence for biometric applications: a survey. International Journal of Computing 15, 40-49.

[8] Donida Labati, R., Genovese, A., Muoz, E., Piuri, V., Scotti, F., Sforza, G., 2016b. Biometric recognition in automated border control: a survey. ACM Computing Surveys 49, 24:1-24:39.

[9] Donida Labati, R., Genovese, A., Piuri, V., Scotti, F., 2014. Touchless fingerprint biometrics: a survey on $2 \mathrm{~d}$ and $3 \mathrm{~d}$ technologies. Journal of Internet Technology 15, 325-332.

[10] Donida Labati, R., Genovese, A., Piuri, V., Scotti, F., 2016c. Toward unconstrained fingerprint recognition: a fully-touchless 3-D system based on two views on the move. IEEE Trans. on Systems, Man, and Cybernetics: Systems 46, 202-219.

[11] Donida Labati, R., Piuri, V., Scotti, F., 2015. Touchless Fingerprint Biometrics. Series in Security, Privacy and Trust, CRC Press.

[12] Donida Labati, R., Scotti, F., 2011. Fingerprint, in: van Tilborg, H., Jajodia, S. (Eds.), Encyclopedia of Cryptography and Security (2nd ed.). Springer, pp. 460-465

[13] Duda, R.O., Hart, P.E., Stork, D.G., 2001. Pattern Classification (2nd Edition). 2 ed., Wiley-Interscience.

[14] Espinoza, M., Champod, C., 2011. Using the number of pores on fingerprint images to detect spoofing attacks, in: Proc. of the Int. Conf. on Hand-Based Biometrics (ICHB), pp. 1-5

[15] Genovese, A., Muñoz, E., Piuri, V., Scotti, F., Sforza, G., 2016. Towards touchless pore fingerprint biometrics: a neural approach, in: Proc. of the 2016 IEEE Congress on Evolutionary Computation (CEC), Vancouver, Canada. pp. 4265-4272.

[16] Goodfellow, I., Bengio, Y., Courville, A., 2016. Deep learning. URL: http://www.deeplearningbook.org. book in preparation for MIT Press.

[17] Haykin, S.O., 2011. Neural Networks and Learning Machines. Pearson Education.

[18] Indovina, M., Hicklin, A., Kiebuzinski, G.I., 2011. ELFT-EFS evaluation of latent fingerprint technologies: extended feature sets [evaluation \# 2]. Technical Report NISTIR 7775. National Institute of Standards and Technology.

[19] Jain, A.K., Chen, Y., Demirkus, M., 2007. Pores and ridges: Highresolution fingerprint matching using level 3 features. IEEE Trans. on Pattern Analysis and Machine Intelligence 29, 15-27.

[20] Jiang, L., Zhao, T., Bai, C., Yong, A., Wu, M., 2016. A direct fingerprint minutiae extraction approach based on convolutional neural networks, in: Proc. of the 2016 Int. Joint Conf. on Neural Networks (IJCNN), pp. 571578.

[21] Johnson, P., Schuckers, S., 2014. Fingerprint pore characteristics for liveness detection, in: Proc. of the Int. Conf. of the Biometrics Special Interest Group (BIOSIG), pp. 1-8.

[22] Kalchbrenner, N., Grefenstette, E., Blunsom, P., 2014. A convolutional neural network for modelling sentences, in: Proc. of the 52nd Annual
Meeting of the Association for Computational Linguistics (ACL), pp. 111.

[23] Ko, K., 2007. User's Guide to NIST Biometric Image Software (NBIS). Technical Report NIST Interagency/Internal Report (NISTIR) - 7392. National Institute of Standards and Technology.

[24] Krizhevsky, A., Sutskever, I., Hinton, G.E., 2012. ImageNet classification with Deep Convolutional Neural Networks, in: Pereira, F., Burges, C.J.C., Bottou, L., Weinberger, K.Q. (Eds.), Advances in Neural Information Processing Systems 25. Curran Associates, Inc., pp. 1097-1105.

[25] Lecun, Y., Bengio, Y., Hinton, G., 2015. Deep learning. Nature 521, 436-444.

[26] Li, D., Kunieda, H., Kumpituck, S., Isshiki, T., 2015. Online detection of spoof fingers for smartphone-based applications, in: Proc. of the IEEE 7th Int. Symp. on High Performance Computing and Communications (HPCC), pp. 1292-1297.

[27] Liu, F., Zhao, Q., Zhang, L., Zhang, D., 2010. Fingerprint pore matching based on sparse representation, in: Proc. of the 2010 20th Int. Conf. on Pattern Recognition (ICPR), pp. 1630-1633.

[28] Liu, N., Li, H., Zhang, M., Liu, J., Sun, Z., Tan, T., 2016a. Accurate iris segmentation in non-cooperative environments using fully convolutional networks, in: Proc. of the 2016 Int. Conf. on Biometrics (ICB), pp. 1-8.

[29] Liu, N., Zhang, M., Li, H., Sun, Z., Tan, T., 2016b. DeepIris: Learning pairwise filter bank for heterogeneous iris verification. Pattern Recognition Letters 82, Part 2, 154-161.

[30] Loy, G., Zelinsky, A., 2003. Fast radial symmetry for detecting points of interest. IEEE Trans. on Pattern Analysis and Machine Intelligence 25, 959-973.

[31] Lu, M., Chen, Z., Sheng, W., 2015. Fingerprint liveness detection based on pore analysis, in: Yang, J., Yang, J., Sun, Z., Shan, S., Zheng, W., Feng, J. (Eds.), Biometric Recognition. Springer International Publishing. volume 9428 of Lecture Notes in Computer Science, pp. 233-240.

[32] Malathi, S., Maheswari, S.U., Meena, C., 2010. Fingerprint pore extraction based on marker controlled watershed segmentation, in: Proc. of the 2nd Int. Conf. on Computer and Automation Engineering (ICCAE), pp. 337-340.

[33] Malathi, S., Meena, C., 2010. An efficient method for partial fingerprint recognition based on local binary pattern, in: Proc. of the IEEE Int. Conf. on Communication Control and Computing Technologies (ICCCCT), pp. 569-572.

[34] Malathi, S., Meena, C., 2011. Improved partial fingerprint matching based on score level fusion using pore and SIFT features, in: Proc. of the Int. Conf. on Process Automation, Control and Computing (PACC), pp. 1-4.

[35] Maltoni, D., Maio, D., Jain, A.K., Prabhakar, S., 2009. Handbook of Fingerprint Recognition. 2nd ed., Springer Publishing Company.

[36] Marcialis, G.L., Roli, F., Tidu, A., 2010. Analysis of fingerprint pores for vitality detection, in: Proc. of the 20th Int. Conf. on Pattern Recognition (ICPR), pp. 1289-1292.

[37] Menotti, D., Chiachia, G., Pinto, A., Schwartz, W.R., Pedrini, H., Falcão, A.X., Rocha, A., 2015. Deep representations for iris, face, and fingerprint spoofing detection. IEEE Trans. on Information Forensics and Security $10,864-879$.

[38] Myronenko, A., Song, X., 2010. Point set registration: Coherent point drift. IEEE Trans. on Pattern Analysis and Machine Intelligence 32, 2262-2275.

[39] Nogueira, R.F., de Alencar Lotufo, R., Machado, R.C., 2016. Fingerprint liveness detection using convolutional neural networks. IEEE Trans. on Information Forensics and Security 11, 1206-1213.

[40] Otsu, N., 1979. A threshold selection method from gray-level histograms. IEEE Trans. on Systems, Man, and Cybernetics 9, 62-66.

[41] Pamplona Segundo, M., de Paula Lemes, R., 2015. Pore-based ridge reconstruction for fingerprint recognition, in: Proc. of the IEEE Conf. on Computer Vision and Pattern Recognition Workshops (CVPRW), pp. 128-133.

[42] Ray, M., Meenen, P., Adhami, R., 2005. A novel approach to fingerprint pore extraction, in: Proceedings of the Thirty-Seventh Southeastern Symposium on System Theory, 2005., pp. 282-286.

[43] Sankaran, A., Dhamecha, T.I., Vatsa, M., Singh, R., 2011. On matching latent to latent fingerprints, in: Proc. of the Int. Joint Conf. on Biometrics (ICB), pp. 1-6.

[44] Saquib, Z., Soni, S., Vig, R., 2010. Sweat pores-based (level 3) novel fingerprint quality estimation, in: Proc. of the 3rd IEEE Int. Conf. on Computer Science and Information Technology (ICCSIT), pp. 525-531. 
[45] Varges da Silva, M., Marana, A.N., Paulino, A.A., 2015. On the importance of using high resolution images, third level features and sequence of images for fingerprint spoof detection, in: Proc. of the IEEE Int. Conf. on Acoustics, Speech and Signal Processing (ICASSP), pp. 1807-1811.

[46] Silva, P., Luz, E., Baeta, R., Pedrini, H., Falcao, A.X., Menotti, D., 2015. An approach to iris contact lens detection based on deep image representations, in: Proc. of the 2015 28th Conf. on Graphics, Patterns and Images (SIBGRAPI), pp. 157-164.

[47] Vatsa, M., Singh, R., Noore, A., Singh, S.K., 2008. Quality induced fingerprint identification using extended feature set, in: Proc. of the 2nd IEEE Int. Conf. on Biometrics: Theory, Applications and Systems (BTAS), pp. 1-6.

[48] Zhang, D., Liu, F., Zhao, Q., Lu, G., Luo, N., 2011. Selecting a reference high resolution for fingerprint recognition using minutiae and pores. IEEE Trans. on Instrumentation and Measurement 60, 863-871.
[49] Zhao, Q., Feng, J., Jain, A.K., 2010a. Latent Fingerprint Matching: Utility of Level 3 Features. Technical Report MSU-CSE-10-14.

[50] Zhao, Q., Jain, A.K., 2010. On the utility of extended fingerprint features: A study on pores, in: Proc. of the IEEE Computer Society Conf. on Computer Vision and Pattern Recognition Workshops (CVPR), pp. 9-16.

[51] Zhao, Q., Liu, F., Zhang, L., Zhang, D., 2010b. Parallel versus hierarchical fusion of extended fingerprint features, in: Proc. of the 2010 20th Int. Conf. on Pattern Recognition (ICPR), pp. 1132-1135.

[52] Zhao, Q., Zhang, D., Zhang, L., Luo, N., 2010c. Adaptive fingerprint pore modeling and extraction. Pattern Recognition 43, 2833-2844.

[53] Zhao, Q., Zhang, L., Zhang, D., Luo, N., 2009. Direct pore matching for fingerprint recognition, in: Tistarelli, M., Nixon, M.S. (Eds.), Advances in Biometrics. Springer Berlin Heidelberg. volume 5558 of Lecture Notes in Computer Science, pp. 597-606. 